\title{
Clinical Research and Clinical Trials
}

\section{Nursing Support Perceived by the Mothers of Hospitalized Children in Bangladesh}

Kohinur Begom MSN¹, Shanzida Khatun PhD, MNSc, RN ${ }^{2}$, Happy Bandana Biswas MNSc, RN³, Mohammad Nurul Anowar PhD, RN ${ }^{4}$

${ }^{1}$ Modernized District Hospital, *Joypurhat

${ }^{2}$ Faculty, Child Health Nursing, *NIANER

${ }^{3}$ Faculty, Child Health Nursing, *NIANER

${ }^{4}$ Faculty, Adult and Elderly Health Nursing, *NIANER

*Corresponding Author: Shanzida Khatun PhD, MNSc, RN, Faculty, National Institute of Advanced Nursing Education and Research, Dhaka.

Received date: May 21, 2021; Accepted date: June 17, 2021; Published date: June 29, 2021

Citation: K Begom, S Khatun, HB Biswas, MN Anowar. (2021) Nursing Support Perceived by the Mothers of Hospitalized Children in Bangladesh. Clinical Research and Clinical Trials. 4(1); DOI: 10.31579/2693-4779/047

Copyright: (c) 2021 Shanzida Khatun. This is an open access article distributed under the Creative Commons Attribution License, which permits unrestricted use, distribution, and reproduction in any medium, provided the original work is properly cited.

\begin{abstract}
Background: Having a child in hospital is a stressful experience for most parents, especially for mothers. In Bangladesh, the number of hospital admission of under-5 children is gradually increasing. Support from nurses can assist mothers to maintain their parenting role and promote quality nursing care.

Objective: The study was conducted to assess the nursing support perceived by the mothers of hospitalized children in Bangladesh.

Methods: A Descriptive cross-sectional study was conducted among 112 mothers whose children were admitted at Dhaka Medical College Hospital. Convenience sampling technique was used to select the study participants. Data was collected by using self-administered structured questionnaires including (1) The Demographic Data Questionnaires and (2) Perception of Nursing Support Questionnaire. Data were analyzed by using descriptive and inferential statistics.

Results: The total mean of nursing support was $3.68(\mathrm{SD}=.25)$. The findings showed that there was a statistically significant difference between mothers'education and nursing support $(\mathrm{F}=2.73, \mathrm{p}=.033)$. There was a statistically significant positive relationship between child age and nursing support $(\mathrm{r}=.22, \mathrm{p}=.019)$. There were statistically significant differences between a child's gender and nursing support of mothers $(\mathrm{t}=1.99, \mathrm{p}=.048)$ which means mothers who had a male child had more nursing support.

Conclusion: The findings of the present study provide information for the nurses 'that help to increase nursing support among mothers of hospitalized children in Bangladesh. Nurses can promote the quality of care and should be aware of the importance of nursing support. Further study is crucial for identifying factors influencing on nursing support perceived by the mothers of hospitalized children. It is important that nurses continue to develop their knowledge about communication and establishing parent support system.
\end{abstract}

Keyword: mothers; perception; nursing support; children

\section{Introduction}

Hospitalization of a child is a stressful experience for parents. About 30\% of children are hospitalized at least once during their childhood and, about $5 \%$ of them are admitted several times, about 5 million children are admitted annually to hospitals for diagnosis or treatment [1].According to Local Health Bulletin (2020), at Dhaka Medical College Hospital total 13,09463 under five children are treated in a year including outpatientdepartment, emergency visits and admission [2].
Children's hospitalization usually leads to disorganization of daily life and family environments, and family members set the child's health and wellbeing as a priority [3].It is shown that this daily life change may be, intensely, considered a hard time for the whole family group and this tends to generate distress [4].Parents experience changes in their parental role when their child is cared for by health care professionals in an unknown environment.The nurse-mothers relationships reach far beyond the stressful experience of hospitalized children and generate more feelings of powerlessness [5]. In order to cope with this stressful 
experience, parents need information about the child's condition, prognosis, treatment plan, and tests. This information allows them to achieve control over situations that produce uncertainty and anxiety [6].By being provided with the appropriate support and the needed information about their child, parents can successfully overcome these challenges and, in turn, provide appropriate support to their children and fulfil their multiple roles within the family.

Nursing support for parents of hospitalized children includes providing a supportive relationship and ongoing information; helping parents maintain their parental role by encouragement, affirmative comment and appraisal; giving emotional support; and providing competent nursing care to the child [7]. Nurses are in a significant position to support parents as they provide care to their sick child, as they are in regular contact with parents during the child's hospitalization [8]. Mothers' psychosocial functioning is important for children's physical and mental health outcomes, and their attitudes during a child's illness, especially during hospitalization, may deeply influence the child's adherence to the care and impact of the disease [9]. A study found that nurses have an important role in assisting parents in defining their role in the relationship between them and their critically ill child.Mok and Leung (2006) found that the highest source of satisfaction for mothers was the support they received from the healthcare team [10].Trask et al (2003) also found that nurses have a crucial role in assisting parents in coping with their child's illness both during hospitalization and discharge [11]. Mother's perception of nursing support is defined as the mother's feeling or view of the nursing care they received from nursing staff during a hospital stay and is acknowledged as an outcome indicator of the quality of nursing care [12].

Nursing support is the most important factor indetermining the child's reaction to nursing care, parents who are frequently informed of the health status of their children by nursing personnel are less likely to have stress and can help their children to adapt better to the hospital environment [13]. During hospitalization, mothers have indicated that they are treated as an outsider, a feeling that can heighten their sense of helplessness and powerlessness [14]. Nurses need to be aware of family members' needs and expectations so that effective support strategies can be implemented, and family satisfaction can be maximized [15].

Effective nursing support strategy is necessary for every parent's satisfaction, especially for mothers of hospitalized children. Although the framework has been identified, little work has been done on the impact of providing the support and no previous research has been performed that has examined nursing support perceived by the mothers with hospitalized children in Bangladesh. In addition, most of the studies were conducted with adifferent culture. Moreover, the findings from the study may provide guidelines for the nurses that can help to provide better nursing support for the mothers on hospitalized children in Bangladesh. That is why it was worth to conduct this phenomenon.

\section{Operational Definition}

\section{Nursing Support}

Nursing support is defined as providing a supportive communication and provision of information related to the the child's illness, treatments, care; enhancing, and supporting the parental role; emotional support to help the parents cope with their own needs related to the childs illness; and caregiving support invoving the quality of care provided to the child $[16,17]$.

In the present study, the perception of nursing support is defined as the perception of mothers regarding to assist mothers by nurse in providing information related to the childs illness, treatment, and care; facilitate safe and consistent care, and provide emotional support to help mothers cope with their own needs to the childs illness. It was measured by the Perception of Nursing Support Questionnaire.

\section{Methods}

\section{Study Design}

This is a descriptive cross-sectional study. It was conducted at Dhaka Medical College Hospital, Dhaka, Bangladesh. The data was collected from December 2019 to February 2020.

\section{Study Participants}

The sample of the study was the mothers of children admitted at Dhaka Medical College Hospital Dhaka, Bangladesh. The study was conducted at the paediatric wards, Dhaka Medical College Hospital (DMCH). This is the biggest tertiary hospital in Bangladesh. This hospital is equipped with 2700 beds, there are 07 pediatric wards/units, including both inpatient and outpatient departments. Therefore, the subjects drawn from this setting was considered to be an appropriate setting. The sample size was estimated by using G-power analysis with the accepted significant level $(\alpha) 0.05$, medium effect size 0.03 , and power 0.80 produced 84 participants. By considering a $20 \%$ attrition rate, the final sample size was 112. A convenience sampling method was used to select the participants. The sample size of the study was 112 with the following inclusion, exclusion criteria. TheInclusion criteria: (1) mothers whose children admitted with chronic illness at Dhaka Medical College Hospital, (2) only mothers, whose children age 3-8 years, (3) understand and speak the Bengali language. The Exclusion criteria:the exclusion criteria were mothers unable to communicate with others.

\section{Instruments}

A self-report structured questionnaire was developed by the researcher based on the literature review. It consists of 2 parts: 1.The Demographic Data Questionnaires, and 2. Perception of Nursing Support Questionnaires.

The Demographic Data Questionnaire: It consists of socio-demographic questionnaires including age, sex, religion, and mother's education, monthly family income, mother's occupation to identify the sociodemographic characteristic of the study participants etc.

Perception of Nursing Support Questionnaire:It was developed by the researcher based on the literature reviewed. This questionnaire consists of 35 items, including three subscales:regarding nursing care (14-items), Nurses behavior (13-items) and communication (8-items). It was a 5point Likert scale; $1=$ strongly disagree to $5=$ strongly agree. A higher score indicated to be better nursing support.

The questionnaire was validated by the three experts: one expert $(\mathrm{PhD}$ Faculty) from NIANER, one assistant professor (nursing) Universal Nursing College, Dhaka, and a paediatrician, Dhaka Medical College Hospital. The original instruments of this study were developed in theEnglish version and translated into a Bengali version and then the back-translated into English based on the back-translation process.

\section{Data Collection Methods}

The study was approved from the Institutional Review Board (IRB) at the National Institute of Advanced Nursing Education and Research (NIANER) and Bangabandhu Sheikh Mujib Medical University (BSMMU), Dhaka. After obtaining permission from the authority of Dhaka Medical College Hospital and signing written informed consents forms by the participants. Distributed questionnaire to an individual participant. Participants were informed aboutho wto fill up the questionnaires without consulting and sharing others. Participants were taken approximately 40 minutes to complete the questionnaires. The primary researcher has checked all return questionnaires for completeness. Anonymity and confidentiality of the respondent were strictly maintained. 


\section{Data Analysis}

Descriptive analysis such as frequency, percentage, mean and standard deviation was used to describe the demographic characteristics of the participants. Inferential analysis including t-test, one way ANOVA and Pearson correlation coefficient were used to describe the relationship between socio-demographic characteristics and perceived nursing support by the participants.

\section{Results}

This chapter represents the findings of the study regarding participant's demographic characteristic and mother's perception of nursing support on hospitalized children. The results of the study are presented under following headlines: (1) Socio-demographic characteristics of mothers and theirchildren, (2) Nursing support perceived by the mothers, (3)
Relationship between socio-demographic characteristic of nursing support perceived by the mothers of hospitalized children.

\section{Socio-Demographic Characteristics of Mothers and Children}

Table 1 shows the frequency, percentage, mean and standard deviation of the socio-demographic characteristics of mothers and their children. The mean age of theparticipated was $29.12(\mathrm{SD}=5.87)$ years. Most of the mothers $(98.2 \%)$ were married. Majority of the mothers $(94.6 \%)$ were Muslim. Most of the mothers $(65.2 \%)$ were lived in rural area. Above half of the mothers $(52.7 \%)$ had primary education. The average monthly family income was 14723.21(SD=7802.99) Taka. Almost all of the mothers $(91.1 \%)$ were housewives. Mean number of children was $2.38(\mathrm{SD}=1.149)$. The mean age of child was $5.97(\mathrm{SD}=1.68)$ years. Most of them were $(61.6 \%)$ male. Around half of the children $(41.1 \%)$ were admitted in pediatric medicineunits.

\begin{tabular}{|c|c|c|c|c|}
\hline Variables & Category & $\mathbf{n}$ & $\%$ & $\mathbf{M}(\mathbf{S D})$ \\
\hline \multicolumn{5}{|c|}{ Mother's characteristics } \\
\hline \multicolumn{2}{|c|}{ Age(years) } & & & $29.12(5.88)$ \\
\hline \multicolumn{5}{|c|}{ Marital status } \\
\hline & Married & 110 & 98.2 & \\
\hline & Others & 2 & 1.8 & \\
\hline \multicolumn{5}{|l|}{ Religion } \\
\hline & Muslim & 106 & 94.6 & \\
\hline & Hindu & 6 & 5.4 & \\
\hline \multicolumn{5}{|l|}{ Residence } \\
\hline & Urban & 39 & 34.8 & \\
\hline & Rural & 73 & 65.2 & \\
\hline \multicolumn{5}{|c|}{ Educational Level } \\
\hline & Non formal schooling & 35 & 31.3 & \\
\hline & Primary & 59 & 52.7 & \\
\hline & SSC & 13 & 11.6 & \\
\hline & HSC & 1 & .9 & \\
\hline & Honors/Degree & 4 & 3.6 & \\
\hline \multicolumn{2}{|c|}{ Monthly family income } & & & $14723.21(7802.99)$ \\
\hline \multicolumn{5}{|l|}{ Occupation } \\
\hline & Housewife & 102 & 91.1 & \\
\hline & Others & 10 & 8.9 & \\
\hline \multicolumn{5}{|c|}{ Children characteristics } \\
\hline \multicolumn{2}{|c|}{ Children Age } & & & $5.97(1.68)$ \\
\hline \multicolumn{5}{|l|}{ Gender } \\
\hline & Male & 69 & 61.6 & \\
\hline & Female & 43 & 38.4 & \\
\hline \multicolumn{2}{|c|}{ Number of children } & & & $2.38(1.149)$ \\
\hline \multicolumn{2}{|c|}{ Admission Ward } & & & \\
\hline & Pediatric surgery & 40 & 35.7 & \\
\hline & Pediatric medicine & 46 & 41.1 & \\
\hline & Others & 26 & 23.2 & \\
\hline
\end{tabular}

Table 1. Distribution of Socio-Demographic Characteristics of the Mothers and their Children (N=112)

\section{NursingSupportPerceived by the Mothers}

Table 2 shows the description of nursing support perceived by the mothers of hospitalized children. The results indicated that the average perception of nursing support (nursing care, behavior, and communication) of mother of hospitalized children was $3.68(\mathrm{SD}=.25)$ in a 5-point Likert scale.

Considering the sub-scale of nursing care the mean was $3.88(\mathrm{SD}=.31)$. Majority of the participants (54.5\%) were strongly agreed on "the nurses being involved in the care and treatment" mean score was $4.52(\mathrm{SD}=.60)$. About $80 \%$ of mothers were agreed on "Nurses taught how to care my baby" with the mean was $3.93(\mathrm{SD}=.65)$. About $75.9 \%$ of mothers were agreed with "Nurses help my baby to adjust with treatment" mean was $3.86(\mathrm{SD}=.86)$. Majority of the mothers $(89.3 \%)$ were agreed on "Nurses prioritize my baby needs" mean score was $3.99(\mathrm{SD}=.45)$. About $87.5 \%$ of participants were agreed "Nurses monitor my baby progress regularly" which mean score was $3.95(\mathrm{SD}=.53)$. "Nurses take measure for potential problems/complications of my baby" ( $89.3 \%)$ mothers were agreed, and mean score was $4.03(\mathrm{SD}=.43)$. About $87.5 \%$ of participants were agreed with "Nurses are aware of changing the treatment plan as doctor suggested" which mean score was $3.99(\mathrm{SD}=.47)$. Majority $(83.9 \%)$ of mothers were agreed to "Nurses allowed me to be involved in my baby's care" which mean was $4.04(\mathrm{SD}=.49)$. 
In termsof mothers' perception regarding nurses behavior, the mean score of behavior was 3.54(SD=.35) among out of 5-point Likert scale. Majority $(88.4 \%)$ of mothers were agreed "Nurses looking smile when they deal with my baby"meanwas $4.06(\mathrm{SD}=.40)$. About $77.7 \%$ of mothers were agreed on the item "Nurses showed concern about my well-being (i.e. sleeping, eating)"mean was 3.79(SD=.79). Majority (82.1\%) of mothers were agreed about" Nurses are sensitive to my baby's special needs" which mean was $3.97(\mathrm{SD}=.45)$. About $87.5 \%$ of mothers were agreed on "Nurses show empathy to my baby "which mean was 3.94(SD=.44). Most of the mothers $(83.9 \%)$ were agreed about "Nurses answered my questions satisfactory" that mean was 3.97(SD=.56). Nurses demonstrating that they did not allow me around my baby, which was showed $(75.0 \%)$ of mothers were agreed that means were $3.63(\mathrm{SD}=.88)$. Around $98.6 \%$ of mothers were agreed on Nurses follow up on delegated task, and mean score was $4.06(\mathrm{SD}=.43)$. Difficulty in getting help from nurses when asked them, themean score was $3.66(\mathrm{SD}=.86)$ showed $76.8 \%$ of mothers were agreed.
In termsof mothers'perception regarding communication, the average score of communication was $3.56(\mathrm{SD}=.33)$. Nurses explaining thing too fast, the mean score was $3.65(\mathrm{SD}=.82)$ mentioned $(78.6 \%)$ of mothers were agreed. About $(87.5 \%)$ mothers were agreed on "Nurses are not talking enough" which mean was 3.79( $\mathrm{SD}=.68)$. Different nurses telling me different (conflicting) things about my baby's condition, mean score was $3.67(\mathrm{SD}=.72)$ and $(77.7 \%)$ mothers were agreed. Majority $(86.6 \%)$ of mothers were agreed on "Nurses explaining me to understand what was being done to my baby (for example: tests, treatments, medicines)" and mean was $4.11(\mathrm{SD}=1.95)$. Around $(74.1 \%)$ of mothers agreed that "Nurses provide information about the things may happen over the next days" mean score was $3.7(\mathrm{SD}=.72)$. More than $(92.9 \%)$ of mothers with "Nurses helped to comfort my baby during or after procedures" mean was 4.02( $\mathrm{SD}=.26)$.

\begin{tabular}{|c|c|c|c|c|c|c|}
\hline \multirow[t]{2}{*}{ Variables } & $\begin{array}{l}\text { Strongly } \\
\text { disagree } \\
(1)\end{array}$ & $\begin{array}{c}\text { Disagree } \\
(2) \\
\end{array}$ & $\begin{array}{c}\text { Uncertainty } \\
\text { (3) } \\
\end{array}$ & $\begin{array}{l}\text { Agree } \\
\text { (4) } \\
\end{array}$ & $\begin{array}{l}\text { Strongly } \\
\text { agree } \\
(5)\end{array}$ & $\mathbf{M}(\mathbf{S D})$ \\
\hline & $\mathbf{n}(\%)$ & $\mathrm{n}(\%)$ & $\mathbf{n}(\%)$ & $\mathrm{n}(\%)$ & $\mathrm{n}(\%)$ & \\
\hline \multicolumn{7}{|l|}{ Nursing Care } \\
\hline Total Mean & & & & & & $3.88(.31)$ \\
\hline $\begin{array}{l}\text { 1.The nurses being involved in the care } \\
\text { and treatment }\end{array}$ & $1(.9)$ & & & $50(44.6)$ & $61(54.5)$ & $4.52(.60)$ \\
\hline $\begin{array}{l}\text { 2.Nurses taught me how to care for my } \\
\text { baby }\end{array}$ & $1(.9)$ & $6(5.4)$ & $4(3.6)$ & $9(80.0)$ & $11(9.8)$ & $3.93(.65)$ \\
\hline $\begin{array}{l}\text { 3. Nurses introduce the names and roles } \\
\text { of the staff caring my baby }\end{array}$ & $2(1.8)$ & $74(66.1)$ & $6(5.4)$ & $(26)(23.2)$ & $4(3.6)$ & $2.61(.98)$ \\
\hline $\begin{array}{l}\text { 4.Nurses help my baby to adjust with } \\
\text { treatment }\end{array}$ & & $9(8.0)$ & $8(7.1)$ & $85(75.9)$ & $10(8.9)$ & $3.86(.68)$ \\
\hline 5.Nurses prioritize my baby needs & $1(.9)$ & $1(.9)$ & $3(2.7)$ & $100(89.3)$ & $7(6.3)$ & $3.99(.45)$ \\
\hline $\begin{array}{l}\text { 6.Nurses monitors my baby progress } \\
\text { regularly }\end{array}$ & & $6(5.4)$ & $1(.9)$ & $98(87.5)$ & $7(6.3)$ & $3.95(.53)$ \\
\hline $\begin{array}{l}\text { 7.Nurses take measure for any potential } \\
\text { problems/complications of my baby }\end{array}$ & & $3(2.7)$ & & $100(89.3)$ & $9(8.0)$ & $4.03(.43)$ \\
\hline $\begin{array}{l}\text { 8.Nurses identify the any health } \\
\text { problem of my baby }\end{array}$ & $1(.9)$ & $42(37.5)$ & $4(3.6)$ & $58(51.8)$ & $7(6.3)$ & $3.25(1.06)$ \\
\hline $\begin{array}{l}\text { 9.Nurses teach me how to administer } \\
\text { the medicine to my child }\end{array}$ & & $1(.9)$ & & $72(64.3)$ & $39(34.8)$ & $4.33(.52)$ \\
\hline $\begin{array}{l}\text { 10.Nurses routinely check my baby's } \\
\text { condition closely }\end{array}$ & & $48(42.9)$ & $6(5.4)$ & $47(42.0)$ & $11(9.8)$ & $3.19(1.10)$ \\
\hline $\begin{array}{l}\text { 11. Nurse notify my child's condition to } \\
\text { the doctor when necessary }\end{array}$ & $1(.9)$ & $1(.9)$ & & $71(63.4)$ & $39(34.8)$ & $4.30(.61)$ \\
\hline $\begin{array}{l}\text { 12.Nurses are aware of changing } \\
\text { thetreatment plan as } \\
\text { doctor suggested }\end{array}$ & $1(.9)$ & $1(.9)$ & $4(3.6)$ & $98(87.5)$ & $8(7.1)$ & $3.99(.47)$ \\
\hline 13.Nurses give medication on time & $1(.9)$ & & & $64(57.1)$ & $47(42.0)$ & $4.39(.59)$ \\
\hline $\begin{array}{l}\text { 14. Nurses allowed me to be involved } \\
\text { in my baby's care }\end{array}$ & & $3(2.7)$ & $2(1.8)$ & $94(83.9)$ & $13(11.6)$ & $4.04(.491)$ \\
\hline \multicolumn{7}{|l|}{ Nurses Behavior } \\
\hline Total Mean & & & & & & $3.54(.35)$ \\
\hline $\begin{array}{l}\text { 15.Nurses looking smile when they } \\
\text { deal with my baby }\end{array}$ & & $2(1.8)$ & & $99(88.4)$ & $11(9.8)$ & $4.06(.40)$ \\
\hline $\begin{array}{l}\text { 16.Nurses showed concern about my } \\
\text { well-being (i.e., sleeping, eating) }\end{array}$ & $4(3.6)$ & $6(5.4)$ & $7(6.3)$ & $87(77.7)$ & $8(7.1)$ & $3.79(.79)$ \\
\hline $\begin{array}{l}\text { 17.Nurses are sensitive to my baby's } \\
\text { special needs }\end{array}$ & & $1(.9)$ & $10(8.9)$ & $92(82.1)$ & $9(8.0)$ & $3.97(.45)$ \\
\hline 18.Nurses show empathy to my baby & $1(.9)$ & & $8(7.1)$ & $98(87.5)$ & $5(4.5)$ & $3.94(.44)$ \\
\hline $\begin{array}{l}\text { 19.Nurses answered my questions } \\
\text { satisfactorily }\end{array}$ & $1(.9)$ & $3(2.7)$ & $4(3.6)$ & $94(83.9)$ & $10(8.9)$ & $3.97(.56)$ \\
\hline $\begin{array}{l}\text { 20.Nurses demonstrating that they did } \\
\text { not allow me around my baby }\end{array}$ & $2(1.8)$ & 19(17.0) & $2(1.8)$ & $84(75.0)$ & $5(4.5)$ & $3.63(.88)$ \\
\hline
\end{tabular}




\begin{tabular}{|c|c|c|c|c|c|c|}
\hline \multirow[t]{2}{*}{ Variables } & $\begin{array}{l}\text { Strongly } \\
\text { disagree } \\
\text { (1) }\end{array}$ & $\begin{array}{c}\text { Disagree } \\
\text { (2) }\end{array}$ & $\begin{array}{l}\text { Uncertainty } \\
\text { (3) }\end{array}$ & $\begin{array}{l}\text { Agree } \\
\text { (4) }\end{array}$ & $\begin{array}{l}\text { Strongly } \\
\text { agree } \\
\text { (5) }\end{array}$ & $\mathbf{M}(\mathbf{S D})$ \\
\hline & $\mathbf{n}(\%)$ & $\mathrm{n}(\%)$ & $\mathbf{n}(\%)$ & $\mathbf{n}(\%)$ & $\mathrm{n}(\%)$ & \\
\hline $\begin{array}{l}\text { 21.Nurses helping me to express my } \\
\text { feelings or concern }\end{array}$ & $1(.9)$ & $49(43.8)$ & $12(10.7)$ & $45(40.2)$ & $5(4.5)$ & $3.04(1.03)$ \\
\hline 22.Nurses follow up on delegated task & & $2(1.8)$ & $1(.9)$ & $97(98.6)$ & $12(10.7)$ & $4.06(.43)$ \\
\hline $\begin{array}{l}\text { 23.Nurses helped me understand my } \\
\text { baby's behavior and reactions }\end{array}$ & $1(.9)$ & $73(65.2)$ & $18(16.1)$ & $14(12.5)$ & $6(5.4)$ & $2.56(.91)$ \\
\hline $\begin{array}{l}\text { 24.Difficulty in getting help from } \\
\text { nurses when asked them }\end{array}$ & $2(1.8)$ & $18(16.1)$ & $1(.9)$ & $86(76.8)$ & $5(4.5)$ & $3.66(.86)$ \\
\hline & & & & & & \\
\hline $\begin{array}{l}\text { 25.Nurses value my experience in } \\
\text { caring my baby }\end{array}$ & $3(2.7)$ & $47(42) 0$. & $17(15.2)$ & $41(36.6)$ & $4(3.6)$ & $2.96(1.02)$ \\
\hline $\begin{array}{l}\text { 26. Nurses made mefeel important for } \\
\text { my baby }\end{array}$ & $3(2.7)$ & $29(25.9)$ & $17(15.2)$ & $58(51.8)$ & $5(4.5)$ & $3.29(.99)$ \\
\hline $\begin{array}{l}\text { 27.Nurses let me know I was doing a } \\
\text { good job in helping my baby }\end{array}$ & $1(.9)$ & $46(41.1)$ & $18(16.1)$ & $42(37.5)$ & $5(4.5)$ & $3.04(1.00)$ \\
\hline \multicolumn{7}{|l|}{ Nurses Communication } \\
\hline Total Mean & & & & & & $3.56(.33)$ \\
\hline 28.Nurses explaining thing too fast & $1(.9)$ & $19(17.0)$ & $1(.9)$ & $88(78.6)$ & $3(2.7)$ & $3.65(.82)$ \\
\hline 29.Nurses are not talking enough & $2(1.8)$ & $9(8.0)$ & $1(.9)$ & $98(87) 5$. & $2(1.8)$ & $3.79(.68)$ \\
\hline $\begin{array}{l}\text { 30.It is difficult to get information } \\
\text { from nurses when I asked them }\end{array}$ & $3(2.7)$ & $93(83.0)$ & $3(2.7)$ & $12(10.71)$ & $1(.9)$ & $2.24(.71)$ \\
\hline $\begin{array}{l}\text { 31.Different nurses telling me different } \\
\text { (conflicting)things about my baby's } \\
\text { condition }\end{array}$ & $2(1.8)$ & $10(8.9)$ & $12(10.7)$ & $87(77.7)$ & $1(.9)$ & $3.67(.72)$ \\
\hline $\begin{array}{l}\text { 32.Nurses explain me to understand } \\
\text { what was being done to my baby (for } \\
\text { example :tests, treatments, medicines) }\end{array}$ & & $4(3.6)$ & $5(4.5)$ & $97(86.6)$ & $1(.9)$ & $4.11(1.95)$ \\
\hline $\begin{array}{l}\text { 33. Nurses include me in discussions or } \\
\text { decisions they made about my baby's } \\
\text { care }\end{array}$ & & $32(28.6)$ & $16(14.3)$ & $60(53.6)$ & $4(3.6)$ & $3.32(.93)$ \\
\hline $\begin{array}{l}\text { 34.Nurses provide information about } \\
\text { the things may happen over the next } \\
\text { days }\end{array}$ & & $13(11.6)$ & $12(10.7)$ & $83(74.1)$ & $4(3.6)$ & $3.70(.72)$ \\
\hline $\begin{array}{l}\text { 35.Nurses helped to comfort my baby } \\
\text { during or after procedures }\end{array}$ & & & $3(2.7)$ & $104(92.9)$ & $5(4.5)$ & $4.02(.26)$ \\
\hline Total mean of nursing support & & & & & & $3.68(.25)$ \\
\hline
\end{tabular}

Table2: Distribution of Nursing Support Perceived by the Mothers of Hospitalized Children (N=112)

3. Relationship between Socio-Demographic Characteristic of the Participants and Nursing Support Perceived by the Mothers of Hospitalized Children

Table 3 shows that relationship between socio-demographic characteristic of nursing support perceived by the mothers of hospitalized children. The results showed that there was a statistically significant relationship between maternal education with nursing support $(\mathrm{F}=2.73, \mathrm{p}=.033)$. It means a mother who has higher education had good perception regarding nursing support. It was also revealed that there was a positive relationship between child age and nursing support perceived by the mothers of hospitalized children $(\mathrm{r}=.22, \mathrm{p}=.019)$. It indicates mothers of comparatively elder children reported significantly higher perceived nursing support than the mother of younger children. There was statistically significant difference between a child's gender and nursing support of mothers $(\mathrm{t}=1.99, \mathrm{p}=.048)$. Considering the gender, it was found that mothers of male children perceived significantly higher nursing support than mothers of female child which means, mothers who had male child had more nursing support than those of female child.

\begin{tabular}{|c|c|c|c|c|c|c|c|c|c|}
\hline \multirow[t]{3}{*}{ Variables } & \multirow[t]{3}{*}{ Category } & \multicolumn{2}{|c|}{ Total Nursing support } & \multicolumn{6}{|c|}{ Nursing support } \\
\hline & & \multirow[b]{2}{*}{$\mathbf{M} \pm \mathbf{S D}$} & \multirow[b]{2}{*}{$\mathbf{t} / \mathbf{F} / \mathbf{r}(\mathbf{p})$} & \multicolumn{2}{|c|}{ Nursing Care } & \multicolumn{2}{|c|}{ Behavior } & \multicolumn{2}{|c|}{ Communication } \\
\hline & & & & $\mathbf{M} \pm \mathbf{S D}$ & $\mathbf{t} / \mathbf{F} / \mathbf{r}(\mathbf{p})$ & $\mathbf{M} \pm \mathbf{S D}$ & $\mathbf{t} / \mathbf{F} / \mathbf{r}(\mathbf{p})$ & $\mathrm{M} \pm \mathrm{SD}$ & t/F/r(p) \\
\hline \multicolumn{2}{|c|}{ Age } & & $.006(0.99)$ & $.029 \pm .70$ & $.029(0.76)$ & $3.54 \pm .34$ & $.12(0.21)$ & $3.56 \pm .33$ & $.003(0.97)$ \\
\hline \multicolumn{2}{|c|}{ Marital status } & & $1.05(0.29)$ & & $.941(0.35)$ & & $.629(0.53)$ & & $.80(0.42)$ \\
\hline & Married & $3.69 \pm .24$ & & $3.89 \pm .31$ & & $3.54 \pm .35$ & & $3.57 \pm .32$ & \\
\hline & Others & $3.50 \pm .10$ & & $3.68 \pm .05$ & & $3.38 \pm .00$ & & $3.38 \pm .53$ & \\
\hline
\end{tabular}




\begin{tabular}{|c|c|c|c|c|c|c|c|c|c|}
\hline \multirow[t]{3}{*}{ Variables } & \multirow[t]{3}{*}{ Category } & \multicolumn{2}{|c|}{ Total Nursing support } & \multicolumn{6}{|c|}{ Nursing support } \\
\hline & & \multirow[b]{2}{*}{$\mathbf{M} \pm \mathbf{S D}$} & \multirow[b]{2}{*}{$\mathbf{t} / \mathbf{F} / \mathbf{r}(\mathbf{p})$} & \multicolumn{2}{|c|}{ Nursing Care } & \multicolumn{2}{|c|}{ Behavior } & \multicolumn{2}{|c|}{ Communication } \\
\hline & & & & $\mathbf{M} \pm \mathbf{S D}$ & $\mathbf{t} / \mathbf{F} / \mathbf{r}(\mathbf{p})$ & $\mathbf{M} \pm$ SD & $\mathbf{t} / \mathbf{F} / \mathbf{r}(\mathbf{p})$ & $\mathbf{M} \pm \mathbf{S D}$ & $\mathbf{t} / \mathbf{F} / \mathbf{r}(\mathbf{p})$ \\
\hline \multicolumn{2}{|c|}{ Religion` } & & $.54(0.58)$ & & $.69(0.49)$ & & $-.09(0.92)$ & & $78(0.43)$ \\
\hline & Muslim & $3.69 \pm .23$ & & $3.89 \pm .29$ & & $3.54 \pm .33$ & & $3.57 \pm 33$ & \\
\hline & Hindu & $3.63 \pm .45$ & & $3.80 \pm .51$ & & $3.55 \pm .60$ & & $3.46 \pm .25$ & \\
\hline \multirow[t]{3}{*}{ Residence } & & & $-1.57(0.12)$ & & $-1.54(0.12)$ & & $-1.36(0.17)$ & & $.72(0.79)$ \\
\hline & Urban & $\begin{array}{c}127.13 \pm \\
9.25\end{array}$ & & $3.82 \pm .34$ & & $3.48 \pm .33$ & & $3.57 \pm 34$ & \\
\hline & Rural & $\begin{array}{c}129.81 \pm \\
8.21\end{array}$ & & $3.92 \pm .28$ & & $3.57 \pm .35$ & & $3.57 \pm .36$ & \\
\hline \multirow[t]{6}{*}{ Education } & & & $2.73(.033)$ & & $1.66(0.16)$ & & $3.29(0.01)$ & & $1.43(0.22)$ \\
\hline & $\begin{array}{c}\text { Non- } \\
\text { formal } \\
\text { schooling }\end{array}$ & $3.68 \pm .22$ & & $3.89 \pm .28$ & & $3.55 \pm .30$ & & $3.55 \pm .22$ & \\
\hline & Primary & $3.68 \pm .25$ & & $3.89 \pm .31$ & & $3.49 \pm .33$ & & $3.61 \pm .39$ & \\
\hline & SSC & $3.64 \pm .21$ & & $3.79 \pm .34$ & & $3.59 \pm .32$ & & $3.44 \pm .23$ & \\
\hline & HSC & $3.20 \pm 0$ & & $3.36 \pm 0$ & & $3.15 \pm 0$ & & $3.00 \pm 0$ & \\
\hline & $\begin{array}{l}\text { Honors/ } \\
\text { Degree }\end{array}$ & $3.99 \pm .29$ & & $4.13 \pm .34$ & & $4.08 \pm .53$ & & $3.59 \pm .18$ & \\
\hline \multicolumn{2}{|c|}{ Monthly Income } & & $-028(0.77)$ & & $.016(0.86)$ & & $.17(0.06)$ & & $-.05(0.57)$ \\
\hline \multicolumn{2}{|c|}{ Occupation } & & $-.20(0.84)$ & & $-1.54(0.12)$ & & $-1.36(0.17)$ & & $.72(0.25)$ \\
\hline & Housewife & $3.68 \pm .25$ & & $3.82 \pm .34$ & & $3.48 \pm .33$ & & $3.55 \pm .26$ & \\
\hline & Others & $3.70 \pm .27$ & & $3.92 \pm .29$ & & $3.57 \pm .35$ & & $3.57 \pm .36$ & \\
\hline \multicolumn{2}{|c|}{ Children age } & & $.22(.019)$ & & $.19(.042)$ & & $.129(0.18)$ & & $.187(.048)$ \\
\hline \multirow[t]{3}{*}{ Gender } & & & $1.99(.048)$ & & $2.42(.017)$ & & $2.50(.014)$ & & $1.65(0.10)$ \\
\hline & Male & $3.72 \pm .27$ & & $3.94 \pm .35$ & & $3.60 \pm .39$ & & $3.52 \pm .24$ & \\
\hline & Female & $3.62 \pm .17$ & & $3.80 \pm .22$ & & $3.44 \pm .22$ & & $3.63 \pm .43$ & \\
\hline \multicolumn{2}{|c|}{$\begin{array}{l}\text { Number of children } \\
\text { Admission ward }\end{array}$} & & $\begin{array}{c}.070(0.46) \\
.13(0.87) \\
\end{array}$ & & $\begin{array}{l}-.045(0.63) \\
.091(0.91) \\
\end{array}$ & & $\begin{array}{c}-.08(0.35) \\
.20(0.81)\end{array}$ & & $\begin{array}{l}.004(0.96) \\
2.37(0.09) \\
\end{array}$ \\
\hline & $\begin{array}{c}\text { Pediatric } \\
\text { surgery }\end{array}$ & $3.69 \pm .24$ & & $3.90 \pm .28$ & & $3.53 \pm .37$ & & $3.59 \pm .22$ & \\
\hline & $\begin{array}{l}\text { Pediatric } \\
\text { medicine }\end{array}$ & $3.67 \pm .27$ & & $3.87 \pm .34$ & & $3.56 \pm .35$ & & $3.49 \pm .26$ & \\
\hline & Others & $3.69 \pm .21$ & & $3.89 \pm .30$ & & $3.51 \pm .29$ & & $3.65 \pm .52$ & \\
\hline
\end{tabular}

Table 3: Relationshipbetween Socio-Demographic Characteristicof the Participants and Nursing Support Perceived by the Mothers of Hospitalized Children $(N=112)$

\section{Discussion}

The present study was conducted withdescriptive cross-sectional study design among mothers whose child was admitted at Dhaka Medical College Hospital, Dhaka and the child aged was 3-8 years. The study aimed is to examine the socio-demographic characteristics and nursing support perceived by the mothers of hospitalized children.

\section{Mothers and Childs Characteristics}

The study has been completed over 112 mothers of hospitalized children. Based on the results, it was found that the mothers received a high level of support (3.68 \pm .25$)$ from the nurses. The result was similar to that of Mok and Leung (2006), who reported an overall mean for received support from nurses of 3.8, and Miles (1999), who reported overall, score $4.1[10]$.

The findings of this study showed that the participants mean age was $29.12(\mathrm{SD}=5.88)$ years. Consistent with other study found that the mothers mean was 28 years [18]. In the context of Bangladesh, the mothers mean age 18.5 years [19]. In the present study, most of the participants were Muslims. According to the Bangladesh Demographics Profile (2020), 89.1\% of people are Muslims [20].

The present study showed above half of the mothers completed primary education. This finding is similar to another study found that the majority of the mothers were completed primary education [21,22]. This result inconsistent with the study of Sanjari et al. (2009)[22].The monthly family income of the participants was 14723.21(SD=7802.99) Taka which was similar to the national standard in Bangladesh, According to Trading Economics global macro models and analysts expectations in Bangladesh is expected to reach $15100.00 \mathrm{BDT} /$ Month by the end of 2020. The result was incongruent with the study conducted in other countries, found that most of the participants family income was insufficient $[21,23]$.

The present study showed that the mean age of child was $5.97(\mathrm{SD}=1.68)$ years. A study Al-Nouri (2005) found that child mean 5years [24]. Incongruent with other studies showed that the mean of a child was 7 years $[21,22,23]$. The present study showed that the majority of the children was a boy, which is similar to the study conducted [25, 22].

\section{Relationship between Socio-Demographic Characteristics of the Participants and Nursing Support Perceived by the Mothers of Hospitalized Children}

There was a statistically significant relationship between demographic characteristics (the mother's education, children age and gender) and nursing support perceived by the mothers of hospitalized children. These results are consistent with other studies [25, 22]. The findings of the present study showed that mothers who had higher education level had greater support than those of mothers had low education 
level $(\mathrm{F}=2.73, \mathrm{P}=.033)$. This finding is similar to the study of Al-Akou, Gharaibeh, and Al-Sallal (2012) found that education influenced mother's perception of nursing support [26]. The finding is also inconsistent with the previous studies showed that mothers' lower education had greater nursing support [22, 27]. This suggests that theeducational level of mothers may be an important predictor affecting their understanding of and concerns related to their children's illness.

In the current study, it was found that there was a significant positive relationship between the child's age and nursing support $(r=.22, p=.019)$ which is congruent study conducted in Turkey showed there was a significant relationship between children age and nursing support [28]. That means mothers who had older child had greater nursing support than those of had younger child. This result is inconsistent with another study found in Iran that there was no significant relationship between children age and nursing support [25].

The present study found that there was a significant relationship between child sex and nursing support $(\mathrm{t}=1.99, \mathrm{p}=.048)$. It means mothers those who had male child have more nursing support. This result suggests that mothers reported more nursing support when their child was a boy. These findings implied that, based on Eastern culture, the gender of the offspring stillmay be important for mothers [22].

\section{Nursing Care}

In terms of sub-dimension of nursing support like nursing care, it was found that there was a statistically significant relationship between demographic characteristic and nursing care. The results found that there was a significant positive relationship between child age and nursing care $(\mathrm{r}=.19, \mathrm{p}=.042)$ which is congruent with the previous study conducted in Turkey [29], which means mothers who had older child had greater nursing care those who had younger child. This result in dissimilar to another study found in Iran [25]. There was a significant differences between child gender and nursing care $(\mathrm{t}=2.42, \mathrm{p}=.017)$. It means mothers those who had male child had better nursing care. This result suggests that mothers reported better nursing care when they have male child. These findings implied that based on Asian culture, the gender of the offspring still may be important for mothers [22,25].

\section{Behavior}

In terms of behavior of nursing support, there was a statistically significant relationship between demographic characteristics and behavior.It was showed that mothers who had ahigher education had better behavior of nurses than those of mothers had alower education level $(\mathrm{F}=3.29, \mathrm{P}=.014)$. This finding is similar to the other study found that education influenced mother's behavior [26,30]. The finding is inconsistent with the previous studies showed that mothers' lower education had better behavior $[22,27]$. The present study found that there was a significant relationship between child sex and nurses behavior $(\mathrm{t}=2.42, \mathrm{p}=.017)$. It means mothers who had male child have better behavior of nurses.

\section{Communication}

In the present study, there was a significant positive relationship between child age and nurses communication $(\mathrm{r}=.19, \mathrm{p}=.42)$ which is congruent with the study conducted in Turkey showed that there was a significant relationship between nurses and child age [29].This result is dissimilar to another study conducted in Iran [25].

On the other hand, the present study did not find any significant differences between mothers' age, marital status, religion, residence, monthly income, occupation, number of child and admission ward.

\section{Limitations}

The instrument of the present study was developed based on the existing literature reviewed and Bangladesh culture and context. In terms of generalizability, the subjects were limited to those mothers admitted to a national hospital in Bangladesh. Therefore, the results may not be generalized to district or rural hospital population.

\section{Conclusion}

The study was adescriptive cross-sectional design carried out from July 2019 to February 2020 at Dhaka Medical College Hospital, Dhaka, Bangladesh. The aim of the study was to the assess nursing support perceived by the mothers of hospitalized children in Bangladesh. The total sample included 112 mothers of children who admitted at Dhaka Medical College Hospital, Dhaka.

The findings of the study showed that the perception of nursing support among mothers of hospitalized children was high $(\mathrm{M}=3.68)$. In terms of relationship, there was a statistically significant relationship between mothers' education, child age, sex, and nursing support perceived by the mothers of hospitalized children. At the same time, other characteristics did not show any significant results. Therefore, it is suggested that nurses may actively participate in facilitating mothers who have ill children in the hospital. Providing support may alleviate mothers' stresses and help to increase their satisfaction regarding pediatric nursing care. Thus, mothers need information about their children situation when they are admitted in hospital.

\section{Recommendations}

Based on the study results and limitations of the present study, the findings of the study can be used in factors identifying nursing support perceived by the mothers of hospitalized children. Further intervention research is also needed to increase better nursing support for mothers of hospitalized children in Bangladesh.

\section{Acknowledgement}

The researchers would like to thanks to all of the mothers who participated in the study and extend their gratitude to Dr. Shanzida Khatun, Chair, Child Health Nursing, NIANER

\section{Competing Interests}

The authors declare that they have no competing interests

\section{References}

1. Reyhani, T., Pourghaznain, T., Mousavi, Z., \&Ghorbani, S. (2014) The effects of presence andguidance of a teacher on the anxiety of hospitalized children. Evidence Based Care, 4, 1522.

2. Local Health Bulletin-2020 (n.d.).

3. Rodrigues, P. F., Amador, D. D., Silva, K. L., Reichert, A. P. S., \&Collet, N. (2013) Interaction between the nursing staff and family from the family's perspective. Esc Anna Nery Rev Enferm, 17, 781-7.

4. Marque, Marques, F. R., Schwartz, E., \&Marcon, S. S. (2014) Experience of mothers on having a child diagnosed and hospitalized by the virus Influenza A (H1N1). Revistabrasileira de enfermagem, 67,220226.

5. Lam, J., Spence, K., \&Halliday, R. (2007) Parents' perception of nursing support in theneonatalintensive care unit (NICU). Neonatal Pediatric and Child Health Nursing, 10,19.

6. Cohen, M. (1995) The Stages of the prediagnostic period in chronic, life-threatening childhood illness: A process analysis. Research in Nursing and Health, 18(1), 39-48.

7. Miles, M. S., Carlson, J., \&Brunssen, S. (1999) The nurse parent support tool. Journalof Pediatric Nursing, 14(1), 44-50.

8. Denney, K. M. (2003) Psychological distress and nursing for latino parents in neonatal intensive care: The contributing 
effects on parenting efficacy. A Dissertation for the degree of Doctor of Philosophy in Education. University of California.

9. Commodari. E. (2010) Children staying in hospital: a research on psychological stress of Caregivers.Italian Journal of Pediatrics, 36(1), 1-9.

10. Mok, E., \& Leung, S. F. (2006) Nurses as providers of support for mothers of premature infants.Journal of Clinical Nursing, 15,726-734.

11. Trask, P. C., Paterson, A. G., Trask, C. L., Bares, C. B., Birt, J., \&Maan, C. (2003) Parent and adolescent adjustment to pediatric cancer: Associations with coping, social support, and family function. Journal of Pediatric Oncology nursing, 20(1),36-47.

12. Gupta, B. S., Shrestha, S., \&Thulung, B. K. (2014) Patient's perception towards qualityNursing care. Journal of Nepal Health Research Council, 12, 83-7.DOI: 10.4172/21671168.1000467

13. Morais, G. S., \& Da Costa, S. F. (2009) Existential experience of mothers of hospitalized children in intensive pediatric care unit. Revista da Escola de Enfermagem da USP, 43, 639-646.

14. Lam, J., Spence, K., \&Halliday, R. (2007) Parents' perception of nursing support in theneonatal intensive care unit (NICU). Neonatal Pediatric and Child Health Nursing, 10,19

15. Malliarou, M., Gerogianni, G., Babatsikou, F., Kotrotsiou, E., \&Zyga, S. (2014) Family perceptions of intensive care unit nurses' roles: a Greek perspective. Health Psychology Research, 2(1),994.

16. House, J. S., Umberson, D., \& Landis, K. R. (1988) Structures and processes of socialsupport.AnnualReview of Sociology, 14(1), 293-318.

17. Miles, M. S., Carlson, J., \&Brunssen, S. (1999) The nurse parent support tool. Journal of Pediatric Nursing, 14(1), 44-50.

18. Jaramillo Santiago, L. X., OsarioGaleano, S. P., \&Salazar Blandon, D.A. (2018) Quality of nursing care:Perception of parents of newborns hospitalized in neonatal units. InvestigacionyEducacionenEfermeria, 36(1),e08.
19. Alam, N., \&Barkat-e-Khuda. (2011) Out-Migration from Matlab-A Rural Area of Bangladesh: Directions, Levels and Differentials. Asian Population Studies, 7(1),35-50.

20. Obaid, K. B. (2015) Psychosocial impact of hospitalization on Ill children in pediatric oncology Wards. Journal of Nurses Health Science.[on-line], 4,72-78. SanjarSanjari, M., Shirazi, F., Heidari, S., Salemi,S.,Rahmani, M., \&Shoghi, M. (2009) Nursing support for parents of hospitalized children.ComprehensivePediatricNursing,32,120 130.

21. Yadav, U., Karn, B. K., Shah, S., \&Chaudhary, R. N. (2015) An investigation into the role of mothers in the care of hospitalized children as perceived by mothers and nurses in pediatric units of BPKIHS, Dharan,Nepal. Health Renaissance, 13,14-21.

22. Al-Nouri, L., \&Basheer, K. (2005) Mothers' perceptions of fever in children. Journal of Tropical Pediatrics, 52, 113-116.

23. Bekmaz, K., Hojjati, H., \&Akhoundzadeh, G. (2019) Relationship between mothers' concerns and nursing support of children admitted to Baqiyatallah Al-Azam Hospital of Ali Abad Katoul, Golestan Province, Iran, in 2018. Modern Care Journal, 16, e92471.

24. Al-Akou,N,A.,Gharaibeh,M.,\&Al-Sallah,R,A. Perception of Jordanian mothers to nursing support during their children hospitalization.Journal of Clinical Nursing,22,233239.

25. Miles, M. S., Burchinal, P., Holditch-Davis, D., Brunssen, S., \& Wilson, S. M. (2002) Perceptions of stress, worry, and support in Black and White mothers of hospitalized, medically fragileinfants. Journal of Pediatric Nursing, 17, 82-88.

26. Kristensson-Hallstrom, I., \&Elander, G. (1997) Parents' experience of hospitalization: different strategies for feeling secure. Pediatric nursing, 23, 361-368.

27. Cimke.S. (2017) Mothers' participation in the hospitalized children's care and their, satisfaction. International Journal of Caring Sciences, 10, 1643-1651.

28. Mitchell, M., Chaboyer, W., Burmeister, E., \& Foster, M. (2009) Positive effects of a nursing intervention on familycentered care in adult critical care. American Journal of Critical Care, 18, 543-552.

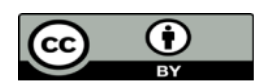

This work is licensed under Creative Commons Attribution 4.0 License

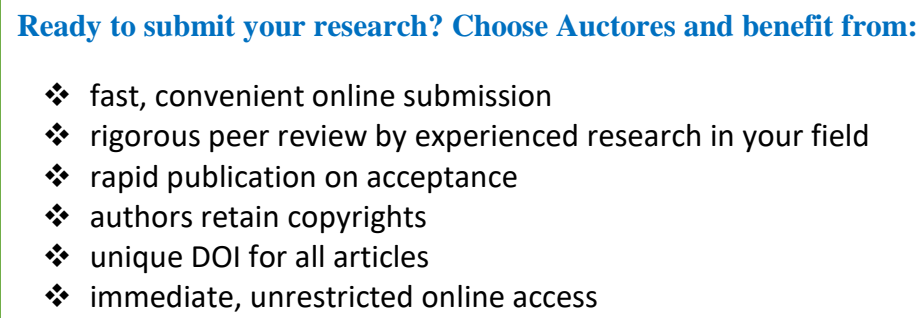

At Auctores, research is always in progress.

Learn more www.auctoresonline.org/journals/clinical-research-andclinical-trials- 